Instituto Internacional de Investigación y Desarrollo Tecnológico Educativo INDTEC, C.A.

DOI: https://doi.org/10.29394/Scientific.issn.2542-2987.2019.4.13.17.322-334

OAI-PMH: http://www.indteca.com/ojs/index.php/Revista Scientific/oai

Ensayo Original / Original Essay

\title{
Transformación del Aprendizaje desde el Enfoque Social (TADES)
}

Autora: Karen Tatiana Quintero Gutiérrez Universidad Pedagógica Experimental Libertador, UPEL

tatica37@hotmail.com

Mérida, Venezuela.

\section{Resumen}

El ensayo pretende cimentar una visión prospectiva sobre la transformación del aprendizaje desde el enfoque social, enfilado desde las teorías sociocultural de Vigotsky (1982) y el interaccionismo simbólico, cuya perspectiva consiste en fomentar un modelo que induzca al sujeto -como ente activo- a transformar su aprendizaje de manera consciente e intencional partiendo de los esquemas de pensamiento construidos a través de la interacción social establecida en el transcurso de su vida, de este modo, promover el aprendizaje fundamentado en el protagonismo del sujeto y orientado a la adquisición de nuevas visiones pedagógicas por parte de los docentes, impulsando la transformación del proceso educativo.

Palabras clave: aprendizaje; interacción social; sistema educativo; pedagogía.

Fecha de Recepción: 12-02-2019
Fecha de Aceptación: 25-05-2019
Fecha de Publicación: 05-08-2019 


\title{
Transformation of Learning from the Social Approach
}

\begin{abstract}
The essay aims to build a prospective vision on the transformation of learning from the social approach, drawn from the sociocultural theories of Vigotsky (1982) and symbolic interactionism, whose perspective is to promote a model that induces the subject -as an active entity- to transform their learning in a conscious and intentional way starting from the thought schemes constructed through the social interaction established in the course of their life, in this way, to promote the learning based on the protagonism of the subject and oriented to the acquisition of new pedagogical visions by teachers, promoting the transformation of the educational process.
\end{abstract}

Keywords: learning; social interaction; education system; pedagogy.

Date Received: 12-02-2019
Date Acceptance: 25-05-2019
Date Publication: 05-08-2019 


\section{Introducción}

La educación, como principal contexto de desarrollo de los individuos, se enfrenta actualmente a cambios sociales que requieren su atención desde las aulas de clase, solicitando abiertamente una renovación en la praxis pedagógica y exigiendo un mayor enfoque en el desarrollo psicosocial del individuo a fin de lograr la transformación educativa necesaria para favorecer la adaptación de éstos a los requerimientos de una sociedad compleja.

En tal sentido, el modelo predominante en la actualidad ha dificultado el desarrollo de las competencias psicosociales que propician la transformación del aprendizaje, por tanto, la praxis pedagógica requiere tomarse de la mano de los enfoques sociales como la teoría sociocultural presentada por Vigotsky y el interaccionismo simbólico a fin de comprender el sistema educativo como un proceso de interacción social.

Bajo el planteamiento expuesto, se puede comprender como la transformación del aprendizaje desde el enfoque social concede luces para instaurar la renovación educativa que tanto se requiere en la actualidad, sugiriendo una aproximación a la sociopedagogía, en tal sentido, se pretende conceptualizar la transformación del aprendizaje y describir los elementos que caracterizan la teoría social, promoviendo la reflexión epistemológica y la práctica analítica en la praxis educativa.

En este orden de ideas el presente ensayo desarrolla los constructos teóricos que permiten comprender la transformación del aprendizaje, así como destaca el aporte de la teoría sociocultural de Vigotsky y del interaccionismo simbólico para este modelo, así como su importancia para la renovación educativa requerida por la sociedad.

\section{Desarrollo}

\subsection{Transformación del aprendizaje}

La transformación del aprendizaje de acuerdo con Abate (2006a): 
refiere a los "procesos de transformación y organización de la información, que ocurren en el sistema cognitivo y que conducen a un proceso de aprendizaje, donde el estudiante puede construir y descubrir el sentido y el significado de la información" (pág. 6).

En este sentido, se puede señalar que en todo proceso de transformación del aprendizaje el estudiante debe establecer relaciones entre los conocimientos que conserva y ha adquirido previamente y la nueva información que se le presenta, transformando su concepción desde la realidad que vive y la manera cómo percibe ésta realidad, para lo cual se hace necesario pensar y reflexionar, construyendo como lo señala Abate (2006b): "una representación interna que se organiza en esquemas mentales, que le permite estructurar los conocimientos, logrando que la información sea adquirida y almacenada en la memoria" (pág. 7).

Por otra parte, se puede señalar que la transformación del aprendizaje se convierte en un proceso constructivo, que parte de establecer vínculos entre la información acopiada en la memoria y la nueva información recibida para alcanzar la cimentación de nuevos significados a través de procesos cognitivos como análisis de información, comparación, organización y almacenamiento.

En tal sentido, Riviere (1987), expone que: "el aprendizaje no se reduce a adquirir respuestas, sino que implica cierto dominio de mecanismos activos de construcción de nociones o proposiciones significativas y la asimilación de éstas a los propios esquemas o conocimientos previos" (pág. 42). Desde esta perspectiva epistemológica, se concibe entonces la transformación del aprendizaje como un proceso sistémico, dinámico y metódico que pone en práctica un conjunto de esquemas cognitivos como percepción, sensación, comparación, procesamiento, argumentación, metamorfosis de la información y construcción de conceptos; para procesar la nueva información y transformarla.

De acuerdo con Abate (2006c), en este proceso: "el estudiante se 
orienta a la comprensión del conocimiento, y para ello, establece relaciones entre la nueva información y los conocimientos ya almacenados" (pág. 8); en consecuencia, hay una innovación tanto del conocimiento como del medio para obtener la información, por ende, se produce una transformación del aprendizaje.

En atención a lo expresado anteriormente, Abate (2006d): llama "esquemas de conocimiento" (pág. 6); al modelo de transformación del aprendizaje, que permite describir cómo aprende un estudiante a través de la transformación de los conocimientos que ya posee, creando un proceso cíclico que parte de la recuperación de la información previamente almacenada en la memoria, para lo cual desarrolla microprocesos de: atención, reconocimiento de patrones semejantes y selección de la información. Seguidamente, se transforma la información ya existente con los conocimientos recientemente adquiridos y se pasa del registro sensorial a la memoria de trabajo (corto plazo) donde son seleccionados los elementos más relevantes para el individuo, luego la información es codificada conceptualmente y almacenada en la memoria a largo plazo.

Interpretando a Pons (2010a): se conciben los esquemas como estructuras de conocimiento cuyo principal propósito reside en almacenar la información construida y relacionarla a través de representaciones internas, permitiéndole al estudiante utilizarla en su proceso de aprendizaje a través de estrategias cognitivas como la selección, recuperación, organización y construcción de una nueva información (pág. 25). Asimismo, es importante acotar que los esquemas ayudan a comprender textos, hechos y acciones, recordar información, clasificar nuevas ideas, adquirir conocimientos nuevos, en conclusión, son elementos que intervienen en el aprendizaje.

En tal sentido, y de acuerdo a los planteamientos de Novak y Gowin (1988): la transformación del aprendizaje, es un proceso cognitivo en el cual se concibe al individuo como "un sujeto consciente de su aprendizaje, 
convirtiéndose en un sujeto más activo, responsable y eficaz frente a los aprendizajes, en definitiva, más capaz de aprender a aprender" (pág. 35).

\subsection{Enfoque Social}

El enfoque social permite comprender como el estudiante construye su aprendizaje a través de la interacción con su medio -su entorno social-, pues la interacción que el individuo establece con la sociedad tiene un rol importante en su funcionamiento intelectual, de allí que, se pueda afirmar que el aprendizaje es, por lo general, un proceso social, tal y como lo plantea Vigotsky (1982a), cuando señala que:

Cada función en el desarrollo cultural del niño aparece dos veces: primero en el nivel social y luego en el individual, primero en medio de otras personas (interpsicológica) y luego dentro del niño (intrapsicológico). Esto aplica igualmente para la atención voluntaria, la memoria lógica y la formación de conceptos. Todas las funciones superiores se originan como relaciones reales entre individuos (pág. 18).

En este orden de ideas, la Teoría Sociocultural, hace hincapié en la participación activa del sujeto con su contexto social, siendo el desarrollo del pensamiento producto de la interacción entre ambos, en tal sentido, Vigotsky (1982b): "el proceso de desarrollo cultural puede definirse en cuanto a su contenido, como el desarrollo de la personalidad del niño y de la concepción del mundo" (pág. 15).

Asimismo, González y Tourón (1992), afianzan esta premisa señalando que: "es en la sociedad donde el individuo adquiere inteligencia, juicio moral y conciencia de sí mismo" (pág. 39); permitiendo establecer la importancia de la interacción social en el progreso pedagógico del sujeto, tal y como lo estudia el interaccionismo simbólico.

De igual manera, se puede afirmar que el desarrollo de los procesos cognitivos del individuo estriban considerablemente de la interacción social 
afirmando que los niños desarrollan su aprendizaje mediante las relaciones que establecen con el medio que los rodea y a partir de allí, van construyendo y reconstruyendo sus conocimientos, adquiriendo nuevas destrezas cognoscitivas, lo cual nos permite aseverar que la transformación del aprendizaje parte del contexto social en el que se desenvuelve el individuo.

En concordancia con lo ya expuesto, se ratifica el enfoque social desde el interaccionismo simbólico, ya que el mismo considera que el ser humano orienta su aprendizaje en función del valor que el nuevo conocimiento tiene para él y lo que este nuevo constructo significa; así mismo, esta fuente de significación es un producto social, que origina una interpretación propia, suponiendo la manipulación de significados, lo cual fundamenta la transformación del aprendizaje.

En tal sentido, el interaccionismo simbólico sostiene que el significado de las cosas constituye para el ser humano un componente de importancia en sí mismo, sin embargo, la base de este radica en el aprendizaje individualizado y autónomo el cual se consolida como una especie de lente en el que se refleja el mundo y a su vez se forma la interacción social.

Igualmente, la capacidad simbólica que tiene la mente humana es en gran medida uno de los principios del interaccionismo simbólico, pues del manejo que se le otorgue a los símbolos, es cuando las personas pueden llegar a construir significados, por tanto, como lo imprime Gil (2007): "el individuo es considerado como un constructor activo de significados, organizados éstos de manera dinámica en torno a procesos compartidos de interacción" (pág. 24); así pues, bajo esta perspectiva el estudiante es capaz de transformar su aprendizaje partiendo de las construcciones sociales que ha internalizado.

Posteriormente, se puede señalar que la teoría del interaccionismo simbólico, asigna preeminencia al mundo social, es decir, a la cultura, la religión, la educación, la familia, entre otros, sin embargo, su principal 
postulado teórico es el acto social, a través del cual se construyen los símbolos que posibilitan la construcción de un nuevo conocimiento a través del desarrollo del pensamiento, así como, la interacción simbólica, ante esto y de acuerdo con Pons (2010b): "concurre en una serie de procesos mentales como la inteligencia reflexiva, la conciencia, las imágenes mentales, el significado y, en términos más generales, la mente" (pág. 25).

En consecuencia, se puede señalar que en el enfoque social sustentado en el interaccionismo simbólico y en la teoría sociocultural, todo ser humano es capaz de reconocer los símbolos sociales y construir o modificar sus significados generando procesos mentales que promueven en el individuo la capacidad de transformar el aprendizaje.

De este modo, el aporte del interaccionismo simbólico y la teoría social a la investigación radica en que permite comprender las interacciones sociales como elemento esencial para la construcción del aprendizaje, vinculación que permite la comprensión de los procesos de transformación y comprensión de los elementos que emergen del individuo en el proceso pedagógico sistémico.

En definitiva, se puede señalar que el enfoque social es de vital importancia en todo proceso educativo, tal y como lo señalan Castellanos y Castro (2017a): "la mejor enseñanza y el aprendizaje más significativo del hombre, proviene del propio contexto en donde hace su vida social" (pág. 78)

\subsection{Transformación del aprendizaje desde el enfoque social (TADES)}

La TADES, trae consigo un enfoque psicopedagógico y complejo, en el cual se induce al sujeto a transfigurar su proceso de aprendizaje partiendo de los esquemas de conocimiento que ha construido a través de las interacciones sociales establecidas en su vida, permitiéndole trazar el camino adecuado que lo llevará a conducir su propio aprendizaje de manera consciente e intencional, de este modo el nuevo aprendizaje estará fundamentado en el protagonismo del sujeto, haciendo del proceso educativo algo meramente personal 
Sin embargo, la actualidad del proceso educativo asevera, tal y como lo expresa Freire (1965): que "muy pocas veces se concibe la educación como praxis, reflexión y acción del hombre sobre el mundo para transformarlo" (pág. 20); de allí que, se considere que los nuevos modelos pedagógicos deben tener presente que para aprender es necesario tomar en cuenta el contexto social en el que se desenvuelve el estudiante, tomando en cuenta que la interacción social del individuo con su entorno establece los pilares fundamentales para que el mismo construya y trasforme su aprendizaje.

De esta manera, la TADES concede especial relevancia al papel que juega el desarrollo social del individuo desde su visión holística, es decir, que toma en cuenta factores morales, éticos, familiares, religiosos, culturales, entre otros, que han sido instaurado en su ser desde el nacimiento y que caracteriza su forma de ver y concebir el mundo y por ende su aprendizaje, tal como lo reseñan Castellanos y Castro (2017b), cuando expresan que: "se adquiere el conocimiento, ideas, actitudes y valores a través de la relaciones interpersonales que se sostiene con la familia y otros miembros para su vida" (pág. 78).

De esta forma, este modelo trae consigo una perspectiva psicopedagógica y compleja, en la cual se induce al sujeto a transformar su aprendizaje desde los esquemas sociales preconcebidos y que son de vital importancia para él, promoviendo una praxis pedagógica centrada en la realidad del individuo.

En correspondencia con lo expuesto anteriormente y en concordancia con Abate (2006e): el modelo TADES, dentro del contexto pedagógico, enfatiza que es el estudiante quien "debe seleccionar la información relevante, comparar las ideas centrales con los conocimientos previos, organizar e integrar estos nuevos conocimientos y también supervisar la comprensión de la información" (pág. 15); construyendo su nuevo aprendizaje de acuerdo a sus intencionalidades personales y generando nuevos esquemas de 
conocimiento, es decir, que se concibe al individuo como sujeto activo en su propio proceso de aprendizaje.

En tal sentido, Lewis y Smith (1980), citados por Olivera (2006), exponen que este modelo: "concibe los individuos como actores existencialmente libres que aceptan, rechazan, modifican o, en cualquier caso, definen las normas, los roles, las creencias, etc. de la comunidad de acuerdo con sus intereses personales y planes del momento" (pág. 85).

En síntesis, el modelo de transformación del aprendizaje desde el enfoque social TADES, se establece como un proceso en el cual el individuo modifica las estructuras conceptuales que ha adquirido previamente a través de la interacción social y aplica los procesos cognitivos necesarios para cimentar las bases de un nuevo aprendizaje, transformando las representaciones ya estructuradas en su memoria y creando nuevos esquemas mentales.

\section{Reflexiones Finales}

El enfoque social se ha vuelto indispensable para la educación y por ende para guiar los procesos de cambio que en ésta área del saber se requieren, es por ello que actualmente las investigaciones y los paradigmas educativos están dirigidos a la profundización de los procesos de enseñanza y de aprendizaje desde la interacción social, al respecto teorías como la social cultural de Vigotsky y el interaccionismo simbólico, consideran al individuo como un ser que adquiere el conocimiento a través de la relación que establece con su entorno.

Partiendo de estos postulados, se ha considerado al individuo como ente activo de su proceso de aprendizaje y no como un mero receptor, pues a través de los conocimientos que ya ha adquirido en su relación con el entorno es capaz de comprender significados y darle un nuevo sentido, transformando de esta manera el aprendizaje ya existente y reconstruyendo uno nuevo. 
De esta manera, la transformación del aprendizaje es producto de un proceso de reflexión, análisis, comprensión y construcción individual del estudiante que parte de unos esquemas preconcebidos en su relación con la sociedad y culmina con la trasformación de ese conocimiento para construir un nuevo significado y por ende un nuevo esquema.

En sintonía con este argumento, la praxis pedagógica en tiempos de cambio, debe concebir la transformación del aprendizaje desde el enfoque social como una herramienta pedagógica que permite atender adecuadamente las demandas de la sociedad, la cual requiere de compromiso ético por parte de los actores educativos y no de la manera cómo se ha venido gestando, únicamente con efectos de transversalidad.

Finalmente, esta visión genera una nueva episteme sobre la transformación del aprendizaje desde el enfoque social, promoviendo de este modo ese cambio innegable que requiere la educación, desentrañando una nueva manera de hacer camino, así también, esta pesquisa, como escenario de creación, transformación y modernización, puede asumir la generatriz del sujeto cognoscente en el sector educativo abordado en torno a las necesidades educativas y en función de aprovechar la innovación del conocimiento, quedando abierta la invitación a construir nuevos derroteros que contribuyan a reconstruir el camino transitado hasta el momento.

\section{Referencias}

Abate, N. (2006a,b,c,d,e). Los desarrollos actuales de la Psicología Cognitiva y sus aportes al proceso de aprendizaje. La Psicología Cognitiva y sus aportes al proceso de aprendizaje. México: Universidad Nacional Autónoma de México - UNAM, págs. 6-18.

Castellanos, E., \& Castro, J. (2017a,b). Estrategias Didácticas para mejorar la Lectura y la Escritura. Revista Scientific, 2(6), 74-91, e-ISSN: 25422987. Recuperado de: 
https://doi.org/10.29394/scientific.issn.2542-2987.2017.2.6.4.74-91

Freire, P. (1965). La Educación como práctica de la Libertad. España: Siglo XXI de España Editores, págs. 1-35.

Gil, M. (2007). Psicología social: Un compromiso aplicado a la salud. 1 ra. Edición, ISBN: 978-84-7733-885-7. Zaragoza, España: Prensas Universitarias de Zaragoza, 491 págs.

González, M. \& Tourón, J. (1992). Autoconcepto y rendimiento escolar. Sus implicaciones en la motivación y en la autorregulación del aprendizaje. ISBN: 84-313-1216-5. Pamplona, España: EUNSA, 484 págs. Recuperado de: http://hdl.handle.net/10171/21388

Novak, J., \& Gowin, D. (1988). Aprendiendo a aprender. ISBN: 9788427029118. Barcelona, España: Ediciones Martínez Roca, 234 págs.

Olivera, E. (2006). La escuela pública como representación simbólica. Una lectura interpretativa desde el interaccionismo simbólico. Diálogos educativos, (11), 81-114, e-ISSN: 0718-1310. Recuperado de:

https://dialnet.unirioja.es/servlet/articulo?codigo $=2122896$

Pons, X. (2010a,b). La aportación a la nueva psicología social del interaccionismo simbólico. Una revisión histórica. Edupsykhé: Revista de Psicología y Psicopedagogía, 9(1), 23-42, e-ISSN: 15790207. Recuperado de:

https://dialnet.unirioja.es/servlet/articulo?codigo=3268858

Riviere, A. (1987). El sujeto de la Psicología cognitiva. Volumen 18 de Alianza psicología, ISBN: 8420665185; ISBN 13: 9788420665184. Madrid, España: Editorial Alianza, 111 págs.

Vigotsky, L. (1982a,b). Historia del desarrollo de las funciones psíquicas superiores. La Habana, Cuba: Editorial Pueblo y Educación, 672 págs. 


\section{Karen Tatiana Quintero Gutiérrez}

e-mail: tatica37@hotmail.com

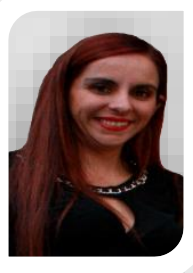

Nacida en Mérida, Venezuela, el 31 de diciembre del año 1978. Profesora en Educación Integral con Distinción Magna Cum Laude de la Universidad Pedagógica Experimental Libertador (UPEL); Postgrado en

Planificación y Evaluación de la Educación en la Universidad Santa María. Docente de educación primaria y Docente de pregrado y postgrado en la UPEL. 